\title{
Article
}

\section{The Impact of Patient Age $\geq 80$ Years on Postoperative Outcomes and Treatment Costs Following Pancreatic Surgery}

\author{
Andreas Andreou ${ }^{\dagger}$, Pauline Aeschbacher ${ }^{+}[$(D), Daniel Candinas and Beat Gloor * \\ Department of Visceral Surgery und Medicine, Inselspital, Bern University Hospital, University of Bern, \\ 3010 Bern, Switzerland; andreas.andreou@insel.ch (A.A.); pauline.aeschbacher@insel.ch (P.A.); \\ daniel.candinas@insel.ch (D.C.) \\ * Correspondence: beat.gloor@insel.ch; Tel.: +41-(0)31-632-76-89 \\ + Authors contributed equally to this manuscript.
}

Citation: Andreou, A.; Aeschbacher, P.; Candinas, D.; Gloor, B. The Impact of Patient Age $\geq 80$ Years on Postoperative Outcomes and Treatment Costs Following Pancreatic Surgery. J. Clin. Med. 2021, 10, 696. https://doi.org/10.3390/jcm10040696

Academic Editor: Yukiyasu Okamura Received: 25 January 2021

Accepted: 7 February 2021

Published: 10 February 2021

Publisher's Note: MDPI stays neutral with regard to jurisdictional claims in published maps and institutional affiliations.

Copyright: (c) 2021 by the authors Licensee MDPI, Basel, Switzerland. This article is an open access article distributed under the terms and conditions of the Creative Commons Attribution (CC BY) license (https:// creativecommons.org/licenses/by/ $4.0 /)$.

\begin{abstract}
As life expectancy is increasing, elderly patients are evaluated more frequently for resection of benign or malignant pancreatic lesions. However, the impact of age on postoperative morbidity, mortality, and treatment costs in octogenarian patients ( $\geq 80$ years) undergoing major pancreatic surgery needs further investigation. The clinicopathological data of patients who underwent pancreatic surgery between January 2015 and March 2019 in a major hepatopancreatobiliary center in Switzerland were assessed. Postoperative outcomes and hospital costs of octogenarians and younger patients were compared in univariate and multivariate regression analysis. During the study period, 346 patients underwent pancreatic resection. Pancreatoduodenectomy, distal pancreatectomy, total pancreatectomy, and other procedures were performed in $54 \%, 20 \%, 13 \%$, and $13 \%$ of patients, respectively. The major postoperative morbidity rate and postoperative mortality rate were $25 \%$ and $3.5 \%$, respectively. A total of 39 patients $(11 \%)$ were $\geq 80$ years old, and 307 patients were $<80$ years old. The majority of octogenarians suffered from ductal adenocarcinoma, whereas among younger patients, other indications for a pancreatic resection were predominant (ductal adenocarcinoma 64\% vs. $41 \%, p=0.006$ ). Age $\geq 80$ was associated with more frequent postoperative medical (pulmonary, cardiovascular) and surgical (high-grade pancreatic fistula, postoperative hemorrhage) complications. Postoperative mortality was significantly higher in octogenarians $(15.4 \%$ vs. $2 \%, p<0.0001)$. This finding may be explained by the higher rate of type C pancreatic fistula (13\% vs. $5 \%$ ), resulting more frequently in postoperative hemorrhage $(18 \%$ vs. $5 \%, p=0.002)$ among patients $\geq 80$ years old. In the multivariate logistic regression analysis, patient age $\geq 80$ years predicted postoperative mortality independently of the tumor entity and surgical technique $(p=0.013$, OR $6.71,95 \% \mathrm{CI}$ [1.5-30.3]). Increased major postoperative morbidity was responsible for lower cost recovery in octogenarians $(94 \%$ vs. $102 \%, p=0.046)$. In conclusion, patient age $\geq 80$ years is associated with increased postoperative medical and surgical morbidity after major pancreatic surgery leading to lower cost recovery and a lower chance for successful resuscitation in patients requiring revisional surgery for postoperative hemorrhage and/or pancreatic fistula. In octogenarian patients suffering from pancreatic tumors, careful selection, and thorough prehabilitation is crucial to achieve the best postoperative and long-term oncologic outcomes.
\end{abstract}

Keywords: pancreatic surgery; elderly; octogenarian; mortality; morbidity; cost recovery

\section{Introduction}

Pancreatic cancer is currently increasing, as is the aging population worldwide. The $\geq 80$ years old population is expected to grow by a factor of four in the next four decades [1].

Pancreatic surgery has significantly evolved over the past decades. Surgical techniques for the pancreas have also been gradually modified to achieve better functional results [2]. Minimally invasive surgery has been introduced in order to minimize surgical trauma [3]. Successive advances in surgical and perioperative management made pancreatic surgery 
safer and significantly reduced postoperative mortality and morbidity [4,5]. As a result of the widened technical possibilities and improved postoperative outcomes in pancreatic surgery, the indication for pancreatic resection has been extended to benign lesions, such as intraductal papillary mucinous neoplasm (IPMN) and chronic pancreatitis [2,6,7]. Moreover, diagnostic tools, such as computed tomography (CT) and magnetic-resonance imaging (MRI), have been increasingly used with enhanced quality and are partially responsible for the growing number of newly found pancreatic lesions [8]. For all these reasons, the number of pancreatic resections performed continues to grow.

Additionally, with the increasing aging of the population, more diagnoses of pancreatic pathologies requiring surgery have been identified in the older population, and even more are expected to be diagnosed in the near future [9-11]. Innovations in surgical, endoscopic, and anesthesiologic treatment, as well as the establishment of modern neoadjuvant systemic therapies, have enabled more patients with advanced age diagnosed with pancreatic ductal adenocarcinoma (PDAC) to undergo surgery. Thus, elderly patients previously considered as unresectable, due to relevant comorbidities or advanced disease are currently more frequently selected as eligible candidates for curative resection [12].

Previous studies investigating age-associated postoperative morbidity and mortality in pancreatic surgery have demonstrated conflicting results. Some single-center studies did not find age as a negative prognostic factor for postoperative outcomes $[8,9,13]$. On the other hand, population-based studies found a negative association between older age and postoperative morbidity and mortality following pancreatic surgery $[11,14,15]$.

Patient age has been associated with higher postoperative complication rates, and surgical morbidity has been related to increased treatment costs in recent studies focusing on pancreatic resections $[16,17]$. Political and hospital administration, as well as health insurance companies, lay great importance on this matter and demand the best possible cost-effectiveness.

As more elderly patients are diagnosed with pancreatic pathologies requiring resection, surgeons need to evaluate operability, taking into account multiple factors, such as concomitant comorbidities, expected perioperative morbidity and mortality, and estimated long-term prognosis in order to optimize the selection of appropriate surgical candidates. We hypothesized that postoperative morbidity and mortality following pancreatic surgery is higher in patients $\geq 80$ years old as compared to younger patients. Consequently, treatment costs are also expected to be higher in the elderly population. Therefore, the aim of this study was to compare postoperative morbidity, mortality, and treatment cost recovery in patients younger and older than 80 years undergoing pancreatic resection in a major hepatopancreatobiliary center in Switzerland.

\section{Material and Methods}

\subsection{Patient Inclusion Criteria}

This study was approved by the Cantonal Ethical Committee of Bern (2019-01171). The anonymity of the patients in this study was preserved at any time during data acquisition and analysis. In this retrospective study [18], all consecutive patients who underwent pancreatic resection for benign and malignant pancreatic lesions between January 2015 and March 2019 at the Department of Visceral Surgery und Medicine, Inselspital, Bern University Hospital were included. Patients $<18$ years old were excluded from the analysis.

\subsection{Preoperative Management}

The standard preoperative patient evaluation included medical history, physical examination, serum laboratory tests, imaging studies, and an anesthesia evaluation. In the case of malignant tumor, the location and extent of tumor burden, as well as the presence of lymph node or distant metastases, were determined by cross-sectional imaging, such as contrast-enhanced CT and/or MRI.

In the case of obstructive jaundice, some patients underwent bile decompression using bile duct stents during endoscopic retrograde cholangiopancreatography, endoscopic 
hepaticogastrostomy, or percutanous transhepatic drainage. The decision to proceed with a bile decompression was individually made according to the duration and extent of cholestasis and the general condition of the patient.

All patients presenting with a malignant tumor were preoperatively discussed at our interdisciplinary tumor board, including pancreatic surgeons, medical oncologists, radiation therapists, and specialized radiologists. Tumor resectability was assessed, and an individualized course of treatment was established for each patient. In patients with borderline resectable or locally-advanced adenocarcinoma, preoperative chemotherapy was recommended, as also described by others [19].

Surgical indication for patients $\geq 80$ years old with pancreatic pathology did not differ from that of younger patients. Medical preconditions and nutritional status [20] were thoroughly evaluated in all patients to identify patients with increased risk for postoperative medical and surgical complications. In the case of relevant comorbidities or nutritional deficiency, preoperative interventions and nutritional prehabilitation were performed to optimize the patients' status prior to surgery. Extensive preoperative counseling was performed, especially with octogenarians, in order to inform patients in detail regarding potential postoperative complications and complicated postoperative clinical course.

\subsection{Surgical Procedure}

Pancreatic resections included pancreatoduodenectomy (PD), distal pancreatectomy, total pancreatectomy, duodenum-preserving pancreatic head resection, and segmental pancreatic resection. Necrosectomies for pancreatitis were excluded. Resections were either performed using a conventional open technique or a laparoscopic-assisted procedure beginning in 2017. Following an upper transverse laparotomy or diagnostic laparoscopy, previously undiagnosed tumor spread into the peritoneum or the liver was ruled out. Classic Kausch-Whipple or pylorus-preserving PD was performed to remove the pancreatic head as previously described [21]. Reconstruction was performed by pancreatojejunostomy using a dissected jejunal loop, end-to-side hepaticojejunostomy, and gastrojejunostomy to re-establish the gastrointestinal passage. Perianastomotic drains were intraoperatively placed to monitor pancreatic fistula, anastomotic leak, and postoperative hemorrhage. In the case of tumor involvement of the portal vein/superior mesenteric vein or superior mesenteric artery, vessel resection, and reconstruction either with anastomosis or graft interposition were performed. Irreversible electroporation (IRE) was used for margin accentuation in patients with a borderline resectable disease to the hepatic artery, celiac trunk, or superior mesenteric artery [22,23].

\subsection{Postoperative Management}

After surgery, all patients were monitored for postoperative complications. Remarkable discharge from perianastomotic drains or persistently increased bilirubin, or lipase levels in drained fluids were indicators for biliary leak and pancreatic fistula, respectively. The criteria of the International Study Group on Pancreatic Fistula (ISGPS) were used for classifying postoperative pancreatic fistulas (POPF) in three severity grades (biochemical leak or A, B, and C), and patients were treated accordingly [24]. Further complications were documented, such as postoperative hemorrhage (defined as hemodynamic- and hemoglobin-relevant bleeding [25]), wound infection (defined as surgical site infection [26]), pulmonary (including pneumonia and pleural effusions), cardiovascular (including hemodynamic instability, cardiac insufficiency, myocardial infarction), and renal complications (including renal failure) [27].

Postoperative morbidity was defined as any complication within 90 days after the surgical procedure and was classified according to a standardized classification [28]. Postoperative complications $\geq$ grade 3 were defined as major morbidity. The postoperative mortality rate was defined as the fraction of patients suffering any complication within 90 days following surgery leading to death. 
Postoperative chemotherapy and radiotherapy were administered in patients based on recommendations of the interdisciplinary tumor board.

\subsection{Statistical Analysis}

The primary endpoint of this study was the comparison between patients $\geq 80$ years old vs. patients $<80$ years regarding postoperative 90 -day mortality. Secondary endpoints were the comparison of the two groups in regard to postoperative 90-day morbidity, cost recovery $(\mathrm{CR})$, defined as the ratio treatment costs/proceeds in percent according to the Swiss diagnosis-related groups (DRG) reimbursement system, and overall-survival (OS). These objectives were investigated in the entire cohort of patients who underwent pancreatic resection during the study period, as well as in subgroups of patients undergoing $\mathrm{PD}$, and patients undergoing resection for PDAC.

Quantitative and qualitative variables were expressed as medians (range) and frequencies. The Chi-square or Fisher's exact test, and the Mann-Whitney $U$ test were used to compare categorical and continuous variables, as appropriate.

To identify factors associated with postoperative mortality after pancreatic resection, clinicopathological variables were analyzed in univariate analysis. In the subsequent multivariate analysis, all factors with $p$ value $<0.05$ in univariate analysis were entered in a logistic regression model to identify independent predictors for postoperative mortality.

Using the Kaplan-Meier method, OS was calculated for patients with PDAC from the date of surgical procedure to the date of death or last follow-up. Log-rank test was used to compare OS between patients $\geq 80$ years old vs. patients $<80$ years old.

$p$ values $<0.05$ were considered statistically significant. For statistical analysis, SPSS software package, version 25, by IBM (Armonk, NY, USA) was used.

\section{Results}

\subsection{Patient Characteristics and Postoperative Outcomes in the Entire Cohort}

From January 2015 to March 2019, 346 patients underwent pancreatic resection, 307 patients were $<80$ years old, and 39 patients $(11 \%)$ were $\geq 80$ years old. In-depth information on the entire cohort and the treatment is given in Table 1. Pancreatic pathologies, including PDAC, periampullary cancers, cystic lesions, neuroendocrine tumors, and chronic pancreatitis, were not significantly differently distributed between patients $\geq 80$ years old and patients $<80$ years old $(p=0.127)$. The major postoperative morbidity rate and 90 -day postoperative mortality rate for the entire cohort were $25 \%$ and $3.5 \%$, respectively. POPF type $C$, postoperative hemorrhage, pulmonary and cardiovascular complications were significantly higher among patients $\geq 80$ years old. Postoperative mortality was also significantly higher among patients $\geq 80$ years old ( $15 \%$ vs. $2 \%, p<0.0001)$. Cost recovery calculation revealed unfavorable outcomes for the older patients (94\% (46-172) vs. 102\% (38-233), $p=0.046$ ). The median hospital costs in our cohort were $38^{\prime} 185$ (15'763-176'318) CHF.

Table 1. Comparison of clinicopathological characteristics of 346 patients undergoing pancreatic surgery according to patient age.

\begin{tabular}{|c|c|c|c|c|}
\hline Variable & $\begin{array}{l}<80 \text { Years } \\
(n=307)\end{array}$ & $\begin{array}{c}\geq 80 \text { Years } \\
(n=39)\end{array}$ & $\begin{array}{l}\text { All Patients } \\
\quad(n=346)\end{array}$ & $p$ Value \\
\hline Gender, $n(\%)$ & & & & 0.106 \\
\hline Female & $139(45)$ & $23(59)$ & $162(47)$ & \\
\hline Male & $168(55)$ & $16(41)$ & $184(53)$ & \\
\hline cost recovery, $\%$, median (range) & $102(38-233)$ & $94(46-172)$ & $101(38-233)$ & 0.046 \\
\hline cardiovascular disease, $n(\%)$ & $157(51)$ & $33(85)$ & $190(55)$ & $<0.0001$ \\
\hline Kidney disease, $n(\%)$ & $41(13)$ & $13(33)$ & $53(15)$ & 0.001 \\
\hline Pulmonary disease, $n(\%)$ & $71(23)$ & $8(21)$ & $79(23)$ & 0.714 \\
\hline Infectious disease, $n(\%)$ & $11(4)$ & $1(3)$ & $12(4)$ & 0.743 \\
\hline Liver disease, $n(\%)$ & $43(14)$ & $7(18)$ & $50(15)$ & 0.510 \\
\hline
\end{tabular}


Table 1. Cont.

\begin{tabular}{|c|c|c|c|c|}
\hline Variable & $\begin{array}{l}<80 \text { Years } \\
(n=307)\end{array}$ & $\begin{array}{c}\geq 80 \text { Years } \\
(n=39)\end{array}$ & $\begin{array}{l}\text { All Patients } \\
\qquad(n=346)\end{array}$ & $p$ Value \\
\hline Neurologic disease, $n(\%)$ & $49(16)$ & $6(15)$ & $55(16)$ & 0.926 \\
\hline Diabetes, $n(\%)$ & $73(24)$ & $11(28)$ & $84(24)$ & 0.544 \\
\hline Genetical alteration, $n(\%)$ & $9(3)$ & $0(0)$ & $9(3)$ & 0.279 \\
\hline BMI, $\mathrm{kg} / \mathrm{m}^{2}$, median (range) & $24(16-46)$ & $24(16-31)$ & $24(16-46)$ & 0.167 \\
\hline $\mathrm{BMI}>30, \mathrm{~kg} / \mathrm{m}^{2}, n(\%)$ & $48(16)$ & $4(19)$ & $52(15)$ & 0.376 \\
\hline Smoking, $n(\%)$ & $131(43)$ & $3(8)$ & $134(39)$ & $<0.0001$ \\
\hline Alcohol consumption, $n(\%)$ & $106(35)$ & $6(15)$ & $112(32)$ & 0.016 \\
\hline ASA status, $n(\%)$ & & & & 0.009 \\
\hline 1 & $7(2)$ & $0(0)$ & $7(2)$ & \\
\hline 2 & $98(32)$ & $5(13)$ & $103(30)$ & \\
\hline 3 & $190(62)$ & $29(74)$ & $219(63)$ & \\
\hline 4 & $12(4)$ & $5(13)$ & $17(5)$ & \\
\hline Resection type, $n(\%)$ & & & & 0.122 \\
\hline Pancreatoduodenectomy & $159(52)$ & $26(67)$ & $185(54)$ & \\
\hline Distal pancreatectomy & $63(21)$ & $6(15)$ & $69(20)$ & \\
\hline Total pancreatectomy & $38(12)$ & $7(18)$ & $45(13)$ & \\
\hline Enucleation & $3(1)$ & $0(0)$ & $3(1)$ & \\
\hline Duodenal-preserving resection & $24(8)$ & $0(0)$ & $24(7)$ & \\
\hline Segmental pancreatic resection & $20(6)$ & $0(0)$ & $20(5)$ & \\
\hline Operating time, $\mathrm{h}$, median (range) & $5.0(1-10)$ & $5.75(3-9)$ & $5.2(1-10)$ & 0.014 \\
\hline Vascular reconstruction, $n(\%)$ & & & & 0.638 \\
\hline none & $240(78)$ & $28(72)$ & $268(78)$ & \\
\hline venous & $56(19)$ & $10(26)$ & $66(19)$ & \\
\hline arterial & $7(2)$ & $1(2)$ & $8(2)$ & \\
\hline combined & $4(1)$ & $0(0)$ & $4(1)$ & \\
\hline Histologic type, $n(\%)$ & & & & 0.006 \\
\hline Adenocarcinoma & $125(41)$ & $25(64)$ & $150(43)$ & \\
\hline other & $182(59)$ & $14(36)$ & $196(57)$ & \\
\hline Pancreatic pathologies, $n(\%)$ & & & & 0.127 \\
\hline PDAC & $125(41)$ & $25(64)$ & $150(43)$ & \\
\hline periampullary malignancies & $46(15)$ & $6(16)$ & $52(15)$ & \\
\hline cystic lesions & $64(21)$ & $5(13)$ & $69(20)$ & \\
\hline neuroendocrine tumors & $28(9)$ & $2(5)$ & $30(9)$ & \\
\hline chronic pancreatitis & $44(14)$ & $1(2)$ & $45(13)$ & \\
\hline Length of ICU stay, days, median (range) & $1(0-37)$ & $1(1-19)$ & $1(0-37)$ & 0.065 \\
\hline Length of hospital stay, days, median (range) & $13(2-87)$ & $13(3-66)$ & $13(2-87)$ & 0.372 \\
\hline Readmission within 90 days, $n(\%)$ & $53(17)$ & $6(17)$ & $59(17)$ & 0.908 \\
\hline Postoperative morbidity, $n(\%)$ & $185(60)$ & $25(64)$ & $210(61)$ & 0.664 \\
\hline Major postoperative morbidity, $n(\%)$ & $73(24)$ & $12(31)$ & $85(25)$ & 0.339 \\
\hline Postoperative mortality, $n(\%)$ & $6(2.0)$ & $6(15.4)$ & $12(3.5)$ & $<0.0001$ \\
\hline POPF, $n(\%)$ & & & & 0.035 \\
\hline None & $221(72)$ & $31(79)$ & $252(73)$ & \\
\hline Type A & $43(14)$ & $3(8)$ & $46(13)$ & \\
\hline Type B & $9(28)$ & $0(0)$ & $28(8)$ & \\
\hline Type C & $15(5)$ & $5(13)$ & $20(6)$ & \\
\hline Re-operation, $n(\%)$ & $42(14)$ & $9(23)$ & $51(15)$ & 0.119 \\
\hline Postoperative hemorrhage, $n(\%)$ & $15(5)$ & $7(18)$ & $22(6)$ & 0.002 \\
\hline Wound infection, $n(\%)$ & $60(20)$ & $8(21)$ & $68(20)$ & 0.886 \\
\hline Pulmonary complication, $n(\%)$ & $34(11)$ & $10(26)$ & $44(13)$ & 0.010 \\
\hline Cardiovascular complication, $n(\%)$ & $15(5)$ & $8(21)$ & $23(7)$ & $<0.0001$ \\
\hline Renal complication, $n(\%)$ & $15(5)$ & $3(8)$ & $18(5)$ & 0.457 \\
\hline
\end{tabular}

BMI, body mass index; ASA, American Society of Anesthesiologists; POPF, postoperative pancreatic fistula; ICU, intensive care unit; PDAC, pancreatic ductal adenocarcinoma. 


\subsection{Comparison of Patients Undergoing Pancreaticoduodenectomy}

In Table 2, data is focusing on the PD, as it is the most challenging of pancreatic resections. PD was performed in 185 patients during the study period, and 26 patients $(14 \%)$ were $\geq 80$ years old. Postoperative mortality was higher in the $\geq 80$ years old group $(11.5 \%$ vs. $1.3 \%, p=0.003)$. There were also more POPF type C, postoperative hemorrhage, and cardiovascular complications in the $\geq 80$ years old group.

Table 2. Comparison of clinicopathological characteristics of 185 patients undergoing pancreatoduodenectomy according to patient age.

\begin{tabular}{|c|c|c|c|c|}
\hline Variable & $\begin{array}{l}<80 \text { Years } \\
(n=159)\end{array}$ & $\begin{array}{c}\geq 80 \text { Years } \\
(n=26)\end{array}$ & $\begin{array}{l}\text { All Patients } \\
\quad(n=185)\end{array}$ & $p$ Value \\
\hline Gender, $n(\%)$ & & & & 0.032 \\
\hline Female & $74(47)$ & $18(69)$ & $92(50)$ & \\
\hline Male & $85(53)$ & $8(31)$ & $93(50)$ & \\
\hline Cost recovery, $\%$, median (range) & $105(42-233)$ & $91(46-172)$ & $102(42-233)$ & 0.045 \\
\hline Heart disease, $n(\%)$ & $82(52)$ & $23(89)$ & $106(57)$ & $<0.0001$ \\
\hline Kidney disease, $n(\%)$ & $21(13)$ & $8(31)$ & $29(16)$ & 0.022 \\
\hline Smoking, $n(\%)$ & $58(37)$ & $1(4)$ & $59(32)$ & 0.001 \\
\hline Alcohol consumption, $n(\%)$ & $54(34)$ & $2(8)$ & $56(30)$ & 0.007 \\
\hline ASA status, $n(\%)$ & & & & 0.123 \\
\hline 1 & $1(1)$ & $0(0)$ & $1(1)$ & \\
\hline 2 & $49(31)$ & $3(12)$ & $52(28)$ & \\
\hline 3 & $102(64)$ & $20(77)$ & $122(66)$ & \\
\hline 4 & $7(4)$ & $3(11)$ & $10(5)$ & \\
\hline Diameter pancreatic duct, mm, median (range) & $4(1-15)$ & $4(3-8)$ & $4(1-15)$ & 0.080 \\
\hline Pancreatic stent intraoperative, $n(\%)$ & $37(24)$ & $1(4)$ & $28(21)$ & 0.021 \\
\hline Histologic type, $n(\%)$ & & & & 0.603 \\
\hline Adenocarcinoma & $83(52)$ & $15(58)$ & $98(53)$ & \\
\hline other & $76(48)$ & $11(42)$ & $87(47)$ & \\
\hline Length of ICU stay, days, median (range) & $1(0-26)$ & $1(1-11)$ & $1(0-26)$ & 0.190 \\
\hline Length of hospital stay, days, median (range) & $13(7-67)$ & $13(3-48)$ & $13(3-67)$ & 0.973 \\
\hline Readmission within 90 days, $n(\%)$ & $26(17)$ & $5(20)$ & $31(17)$ & 0.661 \\
\hline Postoperative morbidity, $n(\%)$ & $86(54)$ & $19(73)$ & 105 (57) & 0.07 \\
\hline Major postoperative morbidity, $n(\%)$ & $36(23)$ & $9(35)$ & $45(24)$ & 0.187 \\
\hline Postoperative mortality, $n(\%)$ & $2(1.3)$ & $3(11.5)$ & $5(2.7)$ & 0.003 \\
\hline POPF, $n(\%)$ & & & & 0.452 \\
\hline None & $123(77)$ & $20(77)$ & $143(77)$ & \\
\hline Type A & $12(8)$ & $2(8)$ & $14(8)$ & \\
\hline Type B & $10(6)$ & $0(0)$ & $10(5)$ & \\
\hline Type C & $14(9)$ & $4(15)$ & $18(10)$ & \\
\hline Re-operation, n (\%) & $24(15)$ & $7(27)$ & $31(17)$ & 0.134 \\
\hline Salvage pancreatectomy, $n(\%)$ & $13(8)$ & $4(15)$ & $17(9)$ & 0.432 \\
\hline Bile leak, $n(\%)$ & $4(3)$ & $0(0)$ & $4(2)$ & 0.414 \\
\hline Gastrointestinal leak, $n(\%)$ & $2(1)$ & $0(0)$ & $2(1)$ & 0.565 \\
\hline Postoperative hemorrhage, $n(\%)$ & $7(4)$ & $5(19)$ & $12(7)$ & 0.004 \\
\hline Wound infection, $n(\%)$ & $34(21)$ & $7(27)$ & $41(22)$ & 0.528 \\
\hline Pulmonary complication, $n(\%)$ & $20(13)$ & $6(23)$ & $26(14)$ & 0.153 \\
\hline Cardiovascular complication, $n(\%)$ & $6(4)$ & $6(23)$ & $12(7)$ & $<0.0001$ \\
\hline Renal complication, $n(\%)$ & $4(3)$ & $2(8)$ & $6(3)$ & 0.167 \\
\hline
\end{tabular}

BMI, body mass index; ASA, American Society of Anesthesiologists; POPF, postoperative pancreatic fistula; ICU, intensive care unit.

\subsection{Comparison of Patients Undergoing Surgery for Pancreatic Ductal Adenocarcinoma}

In Table 3, the focus is on patients suffering from PDAC. This group consisted of 150 patients, and 25 patients (17\%) were $\geq 80$ years old. Postoperative mortality was higher among patients $\geq 80$ years old ( $n=5$ vs. $n=3, p<0.0001$ ). TNM stage and resection margins status were comparable in the two groups. However, the proportion of patients $\geq 80$ years old who received adjuvant chemotherapy was significantly lower in comparison to the $<80$ years cohort $(48 \%$ vs. $79 \%, p=0.001)$. 
Table 3. Comparison of clinicopathological characteristics of 150 patients undergoing resection for pancreatic ductal adenocarcinoma according to patient age.

\begin{tabular}{|c|c|c|c|c|}
\hline Variable & $\begin{array}{l}<80 \text { Years } \\
(n=125)\end{array}$ & $\begin{array}{l}\geq 80 \text { Years } \\
(n=25)\end{array}$ & $\begin{array}{l}\text { All Patients } \\
\quad(n=150)\end{array}$ & $p$ Value \\
\hline Gender, $n(\%)$ & & & & 0.306 \\
\hline Female & $56(45)$ & $14(56)$ & $70(47)$ & \\
\hline Male & $69(55)$ & $11(44)$ & $80(53)$ & \\
\hline Cost recovery, $\%$, median (range) & $103(47-233)$ & $90(46-172)$ & $100(46-233)$ & 0.021 \\
\hline Heart disease, $n(\%)$ & $72(58)$ & $21(84)$ & $93(62)$ & 0.13 \\
\hline Kidney disease, $n(\%)$ & $18(14)$ & $9(36)$ & $27(18)$ & 0.010 \\
\hline Smoking, $n(\%)$ & $48(38)$ & $3(12)$ & $51(34)$ & 0.011 \\
\hline Alcohol consumption, $n(\%)$ & $42(34)$ & $5(20)$ & $47(31)$ & 0.181 \\
\hline ASA status, $n(\%)$ & & & & 0.037 \\
\hline 1 & $2(2)$ & $0(0)$ & $2(1)$ & \\
\hline 2 & $33(26)$ & $1(4)$ & $34(23)$ & \\
\hline 3 & $80(64)$ & $19(76)$ & $99(66)$ & \\
\hline 4 & $10(8)$ & $5(20)$ & $15(10)$ & \\
\hline CA 19-9 preoperative, kU/L, median (range) & $360(2-63690)$ & $311(4-11184)$ & $343(2-63690)$ & 0.692 \\
\hline IRE, $n(\%)$ & $20(16)$ & $2(8)$ & $22(15)$ & 0.302 \\
\hline Operating time, $\mathrm{h}$, median (range) & $6.0(2-9)$ & $6.4(4-9)$ & $6.0(2-9)$ & 0.484 \\
\hline T stage, $n(\%)$ & & & & 0.065 \\
\hline $\mathrm{T} 1$ & $5(4)$ & $0(0)$ & $5(3)$ & \\
\hline $\mathrm{T} 2$ & $45(36)$ & $16(64)$ & $61(41)$ & \\
\hline $\mathrm{T} 3$ & $74(59)$ & $9(36)$ & $83(55)$ & \\
\hline $\mathrm{T} 4$ & $1(1)$ & $0(0)$ & $1(1)$ & \\
\hline N stage, $n(\%)$ & & & & 0.221 \\
\hline N0 & $22(18)$ & $8(32)$ & $30(20)$ & \\
\hline N1 & $74(59)$ & $11(44)$ & $85(57)$ & \\
\hline N3 & $29(23)$ & $6(24)$ & $35(23)$ & \\
\hline Lymph node ratio, median (range) & $\begin{array}{l}3(0-37) / \\
28(6-107)\end{array}$ & $\begin{array}{c}2(0-10) / \\
23(19-62)\end{array}$ & $\begin{array}{c}2(0-37) / \\
28(6-107)\end{array}$ & 0.169 \\
\hline Lymphagiosis carcinomatosa, $n(\%)$ & $25(20)$ & $32(8)$ & $33(22)$ & 0.186 \\
\hline Venous invasion, $n(\%)$ & $16(13)$ & $7(28)$ & $23(15)$ & 0.054 \\
\hline Perineural invasion, $n(\%)$ & $6(5)$ & $2(8)$ & $8(5)$ & 0.516 \\
\hline Tumor differentiation, $n(\%)$ & & & & 0.484 \\
\hline G1 & $18(14)$ & $3(12)$ & $21(14)$ & \\
\hline G2 & $50(40)$ & $14(56)$ & $64(43)$ & \\
\hline G3 & $55(44)$ & $8(32)$ & $63(42)$ & \\
\hline G4 & $2(2)$ & $0(0)$ & $2(1)$ & \\
\hline Tumor margins, $n(\%)$ & & & & 1.0 \\
\hline $\mathrm{R} 1$ & $40(32)$ & $8(32)$ & $48(32)$ & \\
\hline R0 & $85(68)$ & $17(68)$ & $102(68)$ & \\
\hline Length of ICU stay, days, median (range) & $1(0-37)$ & $1(1-19)$ & $1(0-37)$ & 0.269 \\
\hline Length of hospital stay, days, median (range) & $13(2-70)$ & $12(3-66)$ & $13(2-70)$ & 0.667 \\
\hline Readmission within 90 days, $n(\%)$ & $22(18)$ & $3(13)$ & $25(17)$ & 0.571 \\
\hline Postoperative morbidity, $n(\%)$ & $70(56)$ & $15(60)$ & $86(57)$ & 0.713 \\
\hline Major postoperative morbidity, $n(\%)$ & $25(20)$ & $8(32)$ & $33(22)$ & 0.186 \\
\hline Postoperative mortality, $n(\%)$ & $3(2.4)$ & $5(20)$ & $8(5.3)$ & $<0.0001$ \\
\hline POPF, $n(\%)$ & & & & 0.077 \\
\hline None & $104(83)$ & $21(84)$ & $125(83)$ & \\
\hline Type A & $10(8)$ & $1(4)$ & $11(7)$ & \\
\hline Type B & $8(7)$ & $0(0)$ & $8(6)$ & \\
\hline Type C & $3(2)$ & $3(12)$ & $6(4)$ & \\
\hline Re-operation, $n(\%)$ & $17(13)$ & $7(28)$ & $24(16)$ & 0.073 \\
\hline
\end{tabular}


Table 3. Cont.

\begin{tabular}{|c|c|c|c|c|}
\hline Variable & $\begin{array}{c}<80 \text { Years } \\
(n=125)\end{array}$ & $\begin{array}{c}\geq 80 \text { Years } \\
(n=25)\end{array}$ & $\begin{array}{l}\text { All Patients } \\
\quad(n=150)\end{array}$ & $p$ Value \\
\hline Postoperative hemorrhage, $n(\%)$ & $6(5)$ & $6(24)$ & $12(8)$ & 0.001 \\
\hline Wound infection, $n(\%)$ & $30(24)$ & $4(16)$ & $34(23)$ & 0.383 \\
\hline Pulmonary complication, $n(\%)$ & $16(13)$ & $6(24)$ & $22(15)$ & 0.148 \\
\hline Cardiovascular complication, $n(\%)$ & $7(6)$ & $5(20)$ & $12(8)$ & 0.015 \\
\hline Renal complication, $n(\%)$ & $9(7)$ & $2(8)$ & $11(7)$ & 0.889 \\
\hline Neoadjuvant chemotherapy, $n(\%)$ & $13(10)$ & $0(0)$ & $13(9)$ & 0.092 \\
\hline Adjuvant chemotherapy, $n(\%)$ & $99(79)$ & $12(48)$ & $111(74)$ & 0.001 \\
\hline Adjuvant radiotherapy, $n(\%)$ & $1(1)$ & $0(0)$ & $1(1)$ & 0.654 \\
\hline
\end{tabular}

BMI, body mass index; ASA, American Society of Anesthesiologists; IRE, Irreversible electroporation; POPF, postoperative pancreatic fistula; ICU, intensive care unit.

\subsection{Factors Associated with Postoperative Mortality}

Table 4 summarizes the results of the univariate and multivariate analysis of factors associated with 90-day postoperative mortality. Multivariate logistic regression analysis showed that patient age $\geq 80$ years was associated with postoperative mortality, independently of the tumor entity and surgical technique $(p=0.013$, OR $6.71,95 \%$ confidence interval (CI) (1.5-30.3)). Other predictive factors for postoperative mortality were postoperative hemorrhage $(p=0.001$, OR $12.2195 \%$ CI (2.7-55.9)), and renal complications $(p=0.001$, OR 15.80, 95\% CI (3.1-81.4)). Table 5 provides detailed information on the six patients $\geq 80$ years old suffering postoperative 90 -day mortality following pancreatic surgery, including postoperative complications and cause of death. Among the six octogenarians who died after pancreatic surgery, three patients suffered POPF type $C$, and four patients required re-operation because of postoperative hemorrhage. In this group of postoperative mortality, three patients underwent a Whipple procedure, but only two of them suffered POPF. One patient had a soft pancreatic texture, and the other one hard pancreatic texture. Additional to the surgical complications, the cause of death in three patients included respiratory failure (due to aspiration pneumonia), cardiac arrest (due to myocardial infarction), and liver failure (due to thrombosis of the common hepatic artery), respectively (Table 5).

Table 4. Analysis of factors associated with postoperative mortality in 346 patients undergoing pancreatic surgery.

\begin{tabular}{|c|c|c|c|c|c|}
\hline Variable & $\begin{array}{c}\text { Postoperative } \\
\text { Mortality } \\
(n=12)\end{array}$ & $\begin{array}{c}\text { No Postoperative } \\
\text { Mortality } \\
(n=334)\end{array}$ & $\begin{array}{l}\text { All Patients } \\
\quad(n=346)\end{array}$ & $p$ Value & $\begin{array}{c}\text { MV } \\
p \text { Value, OR (CI) }\end{array}$ \\
\hline Male, $n(\%)$ & $7(58)$ & $177(53)$ & $184(53)$ & 0.716 & \\
\hline Age $\geq 80$ years, $n(\%)$ & $6(50)$ & $33(10)$ & $39(11)$ & $<0.0001$ & $\begin{array}{c}0.013 \\
6.71(1.5-30.3)\end{array}$ \\
\hline Heart disease, $n(\%)$ & $8(67)$ & $182(55)$ & $190(55)$ & 0.411 & \\
\hline Kidney disease, $n(\%)$ & $2(17)$ & $52(16)$ & $54(16)$ & 0.918 & \\
\hline Pulmonary disease, $n(\%)$ & $5(42)$ & $74(22)$ & $79(23)$ & 0.114 & \\
\hline Alcohol consumption, $n(\%)$ & $2(17)$ & $110(33)$ & $112(32)$ & 0.237 & \\
\hline ASA status $4, n(\%)$ & $1(8)$ & $16(5)$ & $7(6)$ & 0.577 & \\
\hline Resection type, $n(\%)$ & & & & 0.295 & \\
\hline Pancreatoduodenectomy & $5(42)$ & $180(54)$ & $185(53)$ & & \\
\hline Distal pancreatectomy & $3(25)$ & $66(20)$ & $69(20)$ & & \\
\hline Total pancreatectomy & $4(33)$ & $41(12)$ & $45(13)$ & & \\
\hline Enucleation & $0(0)$ & $3(1)$ & $3(1)$ & & \\
\hline Duodenal-preserving resection & $0(0)$ & $24(7)$ & $24(7)$ & & \\
\hline Segmental pancreatic resection & $0(0)$ & $20(6)$ & $20(6)$ & & \\
\hline
\end{tabular}


Table 4. Cont.

\begin{tabular}{|c|c|c|c|c|c|}
\hline Variable & $\begin{array}{l}\text { Postoperative } \\
\text { Mortality } \\
(n=12)\end{array}$ & $\begin{array}{c}\text { No Postoperative } \\
\text { Mortality } \\
(n=334)\end{array}$ & $\begin{array}{l}\text { All Patients } \\
\quad(n=346)\end{array}$ & $p$ Value & $\begin{array}{c}\text { MV } \\
p \text { Value, OR (CI) }\end{array}$ \\
\hline Histologic type, $n(\%)$ & & & & 0.097 & \\
\hline Adenocarcinoma & $8(67)$ & $142(43)$ & $150(43)$ & & \\
\hline other & $4(33)$ & $192(56)$ & $196(57)$ & & \\
\hline Vascular reconstruction, $n(\%)$ & & & & 0.001 & NS \\
\hline none & $4(34)$ & $264(79)$ & $268(78)$ & & \\
\hline venous & $6(50)$ & $60(18)$ & $66(19)$ & & \\
\hline arterial & $1(8)$ & $7(2)$ & $8(2)$ & & \\
\hline combined & $1(8)$ & $3(1)$ & $4(1)$ & & \\
\hline POPF, $n(\%)$ & & & & 0.036 & NS \\
\hline None & $7(59)$ & $245(73)$ & $252(73)$ & & \\
\hline Type A & $1(8)$ & $45(14)$ & $46(13)$ & & \\
\hline Type B & $1(8)$ & $27(8)$ & $28(8)$ & & \\
\hline Type C & $3(25)$ & $17(5)$ & $20(6)$ & & \\
\hline Postoperative hemorrhage, $n(\%)$ & $6(50)$ & $16(5)$ & $22(6)$ & $<0.0001$ & $\begin{array}{c}0.001 \\
12.21(2.7-55.9)\end{array}$ \\
\hline Wound infection, $n(\%)$ & $3(25)$ & $65(20)$ & $68(20)$ & 0.635 & \\
\hline Pulmonary complication, $n(\%)$ & $6(50)$ & $38(11)$ & $44(13)$ & $<0.0001$ & NS \\
\hline Cardiovascular complication, $n(\%)$ & $5(42)$ & $18(5)$ & $23(7)$ & $<0.0001$ & NS \\
\hline Renal complication, $n(\%)$ & $6(50)$ & $12(4)$ & $18(5)$ & $<0.0001$ & $\begin{array}{c}0.001, \\
15.80(3.1-81.4)\end{array}$ \\
\hline
\end{tabular}

BMI, body mass index; ASA, American Society of Anesthesiologists; MV, multivariate logistic regression analysis; OR, odds ratio; CI, confidence interval; NS, not significant; POPF, postoperative pancreatic fistula.

Table 5. Clinicopathological characteristics of the six patients $\geq 80$ years old suffering postoperative 90 -day mortality following pancreatic surgery.

\begin{tabular}{|c|c|c|c|c|c|c|}
\hline Variable & Patient 1 & Patient 2 & Patient 3 & Patient 4 & Patient 5 & Patient 6 \\
\hline Gender & Male & Male & Female & Male & Female & Female \\
\hline Age & 81 & 85 & 80 & 82 & 81 & 84 \\
\hline Comorbidities & no & cardiac & cardiac & cardiac & renal & cardiac \\
\hline ASA status & 2 & 3 & 3 & 3 & 3 & 3 \\
\hline Type of resection & $\begin{array}{c}\text { Distal } \\
\text { pancreatectomy }\end{array}$ & $\begin{array}{c}\text { Distal } \\
\text { pancreatectomy }\end{array}$ & $\begin{array}{l}\text { Whipple } \\
\text { procedure }\end{array}$ & $\begin{array}{l}\text { Whipple } \\
\text { procedure }\end{array}$ & $\begin{array}{l}\text { Whipple } \\
\text { procedure }\end{array}$ & $\begin{array}{c}\text { total } \\
\text { pancreatectomy }\end{array}$ \\
\hline $\begin{array}{c}\text { Pancreatic } \\
\text { anastomosis }\end{array}$ & - & - & $\begin{array}{l}\text { pancreato- } \\
\text { jejunostomy }\end{array}$ & $\begin{array}{l}\text { pancreato- } \\
\text { jejunostomy }\end{array}$ & $\begin{array}{l}\text { pancreato- } \\
\text { jejunostomy }\end{array}$ & - \\
\hline Pancreas texture & soft & soft & hard & soft & hard & soft \\
\hline $\begin{array}{l}\text { Pancreatic duct } \\
\text { diameter (mm) }\end{array}$ & - & - & 8 & 3 & 4 & - \\
\hline POPF & type A & type C & no & type C & type C & no \\
\hline $\begin{array}{l}\text { Postoperative } \\
\text { hemorrhage }\end{array}$ & no & yes & yes & yes & no & yes \\
\hline Re-operation & no & yes & yes & yes & yes & yes \\
\hline $\begin{array}{l}\text { Other } \\
\text { postoperative } \\
\text { complications }\end{array}$ & $\begin{array}{l}\text { myocardial } \\
\text { infarction }\end{array}$ & $\begin{array}{l}\text { pneumonia, } \\
\text { acute kidney } \\
\text { failure }\end{array}$ & $\begin{array}{l}\text { thrombosis of } \\
\text { common hepatic } \\
\text { artery }\end{array}$ & $\begin{array}{c}\text { aspiration } \\
\text { pneumonia }\end{array}$ & $\begin{array}{c}\text { aspiration } \\
\text { pneumonia, acute } \\
\text { kidney failure }\end{array}$ & $\begin{array}{l}\text { pneumonia, acute } \\
\text { kidney failure }\end{array}$ \\
\hline ICU stay, days & 8 & 19 & 3 & 4 & 7 & 11 \\
\hline $\begin{array}{l}\text { Postoperative day } \\
\text { of death }\end{array}$ & 18 & 67 & 3 & 22 & 11 & 52 \\
\hline Cause of death & cardiac arrest & sepsis & liver failure & respiratory failure & sepsis & sepsis \\
\hline
\end{tabular}




\subsection{Long-Term Survival of Patients with PDAC}

Among patients with PDAC, patient age was not associated with oncologic outcome using a cut-off value of 80 years. Three-year overall survival rates were comparable between patients $<80$ years old and patients $\geq 80$ years old $(42.4 \%$ vs. $41.9 \%, p=0.059$, Figure 1$)$. The median survival was $24(18-30)$ months for patients $<80$ years old vs. $13(6-20)$ months for patients $\geq 80$ years old $(p=0.059)$.

We analyzed the impact of sex on OS in the subgroups of patients $<80$ years old and patients $\geq 80$ years old. We found no statistically significant survival difference between female and male patients, both in patients $<80$ years old (female, $n=56$, 3-year OS $45 \%$ vs. male, $n=68$, 3-year OS $40.8 \%, p=0.638$ ) and patients $\geq 80$ years old (female, $n=14$, 3 -year OS $51.6 \%$ vs. male, $n=11$, 3-year OS 31.8\%, $p=0.571$ ), even though OS was higher for female patients in both groups.

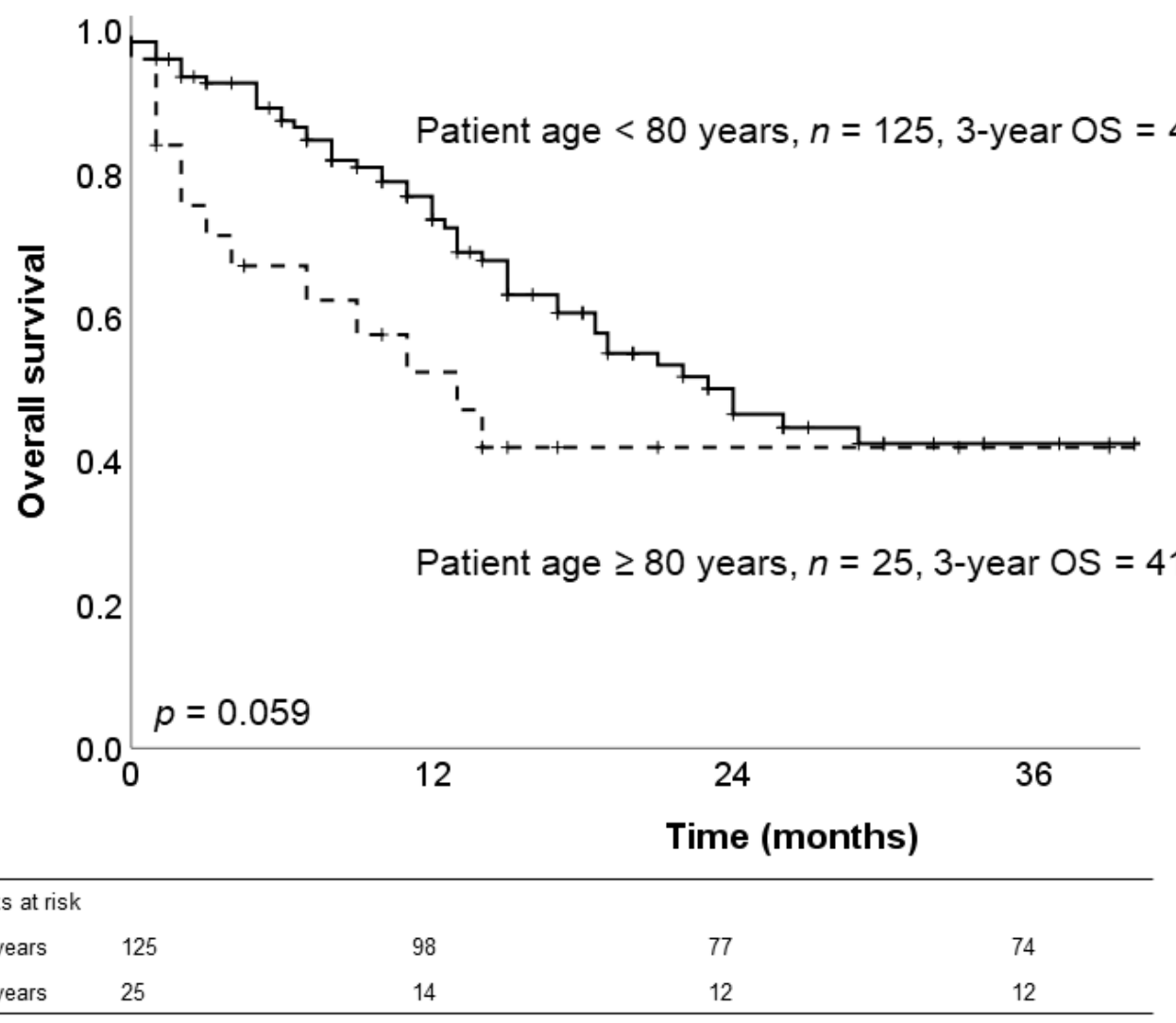

Figure 1. Comparison of overall survival (OS) in patients with pancreatic ductal adenocarcinoma $(n=150)$ according to patient age ( $<80$ years vs. $\geq 80$ years).

\section{Discussion}

In an unselected group of 346 consecutive patients, our study examined the impact of patient age on postoperative mortality and morbidity after pancreatic resective surgery. Importantly, we focused not only on the entire cohort, but also on the group of patients undergoing PD and on those suffering from PDAC.

Patient age $\geq 80$ years was significantly associated with a higher rate of both surgical and medical complications in our study. Additionally, the postoperative mortality rate was higher in our cohort of patients $\geq 80$ years old. These results are comparable to those of previous studies, including a large study from Germany, investigating the results of 1705 pancreatic operations, including $76(4.5 \%)$ patients $\geq 80$ years old [8]. A recent international multicentric study with 3624 elderly patients from Belgium, the Netherlands, and Norway showed postoperative mortality rates beyond $10 \%$ for patients $\geq 80$ years old, underlining the importance of investigating the role of patient age on outcomes following 
pancreatic surgery [15]. The aim of this study was not to determine a cut-off age for pancreatic surgery, but to underline the importance of correct identification of the candidates who will benefit from surgery the most, despite advanced age. Elderly patients are more fragile and have fewer capacities to overcome major postoperative complications [29].

A higher rate of medical complications, such as pneumonia and cardiovascular adverse events, has been found in the cohort of patients $\geq 80$ years old in our study. Elderly patients presented more frequently with preoperative cardiovascular and pulmonary comorbidities, which may have deteriorated postoperatively. The association between relevant comorbidities and increased postoperative complications has been previously discussed [30]. In this regard, frailty among elderly patients is known as a state of decreased capacity to cope with stress factors in order to restore homeostasis [1]. However, frailty is an independent factor that does not always correlate with age and may also be reversible [31]. Our results, indicating that the elderly population is more vulnerable to postoperative complications with a lower capacity to recover, underline the need for precise preoperative assessment of medical preconditions in order to improve patient selection and reduce the risk for selected patients. Patient-level factors contributed the most to the increased risk of mortality after PD in a recent US study [32]. Additionally, interventions must be undertaken prior to surgery to improve nutritional status [20], and the medical comorbidities, when possible. It is important to mention that we have conducted even more extensive conversations with the older patients, mostly together with their families, in order to find out how their physical capacity in daily life was and how much treatment they wished to undergo. Based on the results of our study showing that patient age is a prognostic factor for adverse events after surgery, elderly patients and their close family members were made aware of this risk during preoperative counseling. They were explicitly informed of the possible need for prolonged intensive care, respiratory, cardiovascular, and renal support, in the case of a complication. If, during the preoperative counseling, life-support measures were denied by the patient in the case of a postoperative complication, the indication for major resection was restricted.

ASA status has been identified as a useful tool for preoperative assessment [33]. We showed that the group of patients $\geq 80$ years old had mostly higher ASA scores, however ASA status of 4 was not associated with higher postoperative mortality in our study. Nevertheless, this result may be associated with the low number of ASA 4 patients, and octogenarians with an ASA status 4 are not considered as surgical candidates in our practice anymore.

Patients $\geq 80$ years old suffered more frequently from POPF type $C$ with subsequent hemorrhage [34]. Increased patient age is only one factor among others, such as BMI, visceral fat thickness, pancreatic duct size, and pancreas tissue texture that have been previously shown to be associated with POPF [35-37]. Thus, postoperative hemorrhage, due to vessel erosion, may have been responsible for the increased postoperative mortality in the octogenarian cohort, additionally to the increased preoperative risk factors. Patient age $\geq 80$ years was associated with postoperative mortality in the entire cohort, as well as in the subset of patients undergoing pancreaticoduodenectomy, and patients with PDAC.

Postoperative mortality among octogenarians was even higher in patients with PDAC compared to other indications for pancreatic surgery. This difference may be explained by the fact that oncological patients often present with more severe malnutrition and have less capacity to recover [38]. Moreover, even though not statistically significant, there was a trend to more total pancreatectomies among octogenarians. This is in concordance with previous studies as total pancreatectomy is often favored over a partial pancreaticoduodenectomy in high-risk patients with more advanced disease [39]. Nevertheless, patient age was associated with postoperative mortality independently of the tumor entity and surgical technique in the multivariate analysis. However, the large confidence interval (1.5-30.3) for postoperative mortality in the multivariate analysis indicates the relatively small number of patients involved. 
Cost recovery following pancreatic resection was worse for patients $\geq 80$ years old in the entire cohort, as well as in patients with PDAC and patients undergoing PD. Higher surgical ( $\mathrm{POPF}$, hemorrhage) and medical (pulmonary, cardiovascular) postoperative complications in this cohort have contributed to higher treatment costs, and thus, worse financial balance. Previous studies confirmed that higher costs are directly related to the presence of severe complications and longer hospital stay [17]. Grade III and IV complications cause a doubling of the costs compared to an uneventful postoperative course [17]. Nevertheless, financial criteria and potentially worse cost recovery after surgery are currently not taken into consideration, when evaluating octogenarians or any other patient cohort for pancreatic resection or other oncologic treatment.

In our study, we have observed that the older population was less likely to receive adjuvant chemotherapy. Postoperative complications (especially pancreatic fistula and postoperative hemorrhage), but also higher age, and lower center volume were predictive factors for not receiving adjuvant chemotherapy after pancreatic resection in a recent Dutch multicentric study [40]. In our cohort, this result can be explained by higher rates of POPF and postoperative hemorrhage among patients $\geq 80$ years old. Additionally, a large proportion of our patients undergoes systemic treatment in peripheral hospitals, where older patients are often treated less aggressively, and higher dropout rates during adjuvant chemotherapy are observed [41]. In our center, we have been increasingly recommending neoadjuvant chemotherapy for 3-6 months for patients with borderline resectable or locallyadvanced PDAC in the most recent years. Among 150 patients with PDAC, only 13 patients $<80$ years were administered preoperative chemotherapy in our study. Due to our relatively strict selection criteria for pancreatic surgery among elderly patients, patients $\geq 80$ years old with such extended tumor burden requiring long preoperative chemotherapy and complex surgery were mostly not appropriate surgical candidates. Additionally, elderly patients $\geq 80$ years old often did not qualify for the preferred regimen with FOLFIRINOX, which has a relevant toxicity profile, as indicated by the study from Conroy et al., which included only patients $<80$ years in the FOLFIRINOX arm [42].

Our survival analysis for patients with PDAC showed that patient age was not significantly associated with oncologic outcome. Although, mortality has been more frequently observed among patients $\geq 80$ years old in the first two years following oncologic resection, survival did not differ between the two groups on the long-term and 3-year OS was identical ( $<80$ years: $42.4 \%$ vs. $\geq 80$ years: $41.9 \%, p=0.059$ ). This result indicates that prolonged survival is possible for well-selected patients $\geq 80$ years old, who undergo oncologic resection with an uneventful postoperative course, enhanced recovery, and fast return to normal function.

Finally, our study has several limitations. It is a retrospective analysis of an inhomogeneous population of patients, including benign and malignant pancreatic lesions. However, it represents the standard case profile of a high-volume center in Switzerland, reflecting our experience with an octogenarian population. We have performed analysis using the cut-off age of 80 years, acknowledging that results may slightly vary by choosing another value. However, our aim was not to provide evidence on whether to operate on patients $\geq 80$ years old or not, but rather to indicate the challenges older and multimorbid patients requiring pancreatic surgery provide and to discuss ways to improve outcomes in this cohort.

\section{Conclusions}

Patient age of $\geq 80$ years was associated with a higher risk for medical and surgical postoperative complications and mortality following pancreatic surgery. Reduced capacity for successful resuscitation and postoperative recovery when requiring revision surgery among the elderly patient cohort has been identified. Surgical treatment of octogenarians was also related to a lower cost recovery rate. Nevertheless, pancreatic surgery remains the only curative-intended approach for pancreatic cancer, and therefore, careful patient selection is essential to achieve favorable outcomes. 
Author Contributions: Conceptualization, A.A., P.A., D.C., B.G.; methodology, A.A., P.A., D.C., B.G.; software, A.A., P.A., D.C., B.G.; validation, A.A., P.A., D.C., B.G.; formal analysis, A.A., P.A., D.C., B.G.; investigation, A.A., P.A., D.C., B.G.; resources, A.A., P.A., D.C., B.G.; data curation, A.A., P.A., D.C., B.G.; writing—original draft preparation, A.A., P.A., D.C., B.G.; writing-review and editing, A.A., P.A., D.C., B.G.; visualization, A.A., P.A., D.C., B.G.; supervision, A.A., P.A., D.C., B.G.; project administration, A.A., P.A., D.C., B.G. Part of the data in this manuscript was presented at the 106th Annual Congress of the Swiss Surgical Association, May 2019, Bern, Switzerland. All authors have read and agreed to the published version of the manuscript.

Funding: This research received no external funding.

Institutional Review Board Statement: The study was conducted according to the guidelines of the Declaration of Helsinki, and approved by the Cantonal Ethical Committee of Bern (2019-01171, 6 August 2019).

Informed Consent Statement: Patient consent was waived by the Cantonal Ethical Committee of Bern (2019-01171).

Conflicts of Interest: The authors declare no conflict of interest.

\section{References}

1. Clegg, A.; Young, J.; Iliffe, S.; Rikkert, M.O.; Rockwood, K. Frailty in elderly people. Lancet 2013, 381, 752-762. [CrossRef]

2. Balcom, J.H.R.D., 4th; Warshaw, A.L.; Chang, Y.; Fernandez-del Castillo, C. Ten-year experience with 733 pancreatic resections: Changing indications, older patients, and decreasing length of hospitalization. Arch Surg. 2001, 136, 391-398. [CrossRef] [PubMed]

3. Palanivelu, C.; Takaori, K.; Abu Hilal, M.; Kooby, D.A.; Wakabayashi, G.; Agarwal, A.; Berti, S.; Besselink, M.G.; Chen, K.H.; Gumbs, A.A.; et al. International Summit on Laparoscopic Pancreatic Resection (ISLPR) "Coimbatore Summit Statements". Surg. Oncol. 2018, 27, A10-A15. [CrossRef]

4. Cameron, J.L.; He, J. Two Thousand Consecutive Pancreaticoduodenectomies. J. Am. Coll. Surg. 2015, 220, 530-536. [CrossRef]

5. van Rijssen, L.B.; Zwart, M.J.; van Dieren, S.; de Rooij, T.; Bonsing, B.A.; Bosscha, K.; van Dam, R.M.; van Eijck, C.H.; Gerhards, M.F.; Gerritsen, J.J.; et al. Variation in hospital mortality after pancreatoduodenectomy is related to failure to rescue rather than major complications: A nationwide audit. HPB 2018, 20, 759-767. [CrossRef] [PubMed]

6. Tanaka, M. Clinical Management and Surgical Decision-Making of IPMN of the Pancreas. Methods Mol. Biol. 2019, 1882, 9-22. [PubMed]

7. Schnelldorfer, T.; Lewin, D.N.; Adams, D.B. Operative Management of Chronic Pancreatitis: Longterm Results in 372 Patients. J. Am. Coll. Surg. 2007, 204, 1039-1045. [CrossRef]

8. Belyaev, O.; Herzog, T.; Kaya, G.; Chromik, A.M.; Meurer, K.; Uhl, W.; Müller, C.A. Pancreatic Surgery in the Very Old: Face to Face With a Challenge of the Near Future. World J. Surg. 2013, 37, 1013-1020. [CrossRef]

9. Makary, M.A.; Winter, J.M.; Cameron, J.L.; Campbell, K.A.; Chang, D.; Cunningham, S.C.; Riall, T.S.; Yeo, C.J. Pancreaticoduodenectomy in the Very Elderly. J. Gastrointest. Surg. 2006, 10, 347-356. [CrossRef]

10. Wu, W.; He, X.; Yang, L.; Wang, Q.; Bian, X.; Ye, J.; Li, Y.; Li, L. Rising trends in pancreatic cancer incidence and mortality in 2000-2014. Clin. Epidemiol. 2018, 10, 789-797. [CrossRef]

11. Riall, T.S.; Reddy, D.M.; Nealon, W.H.; Goodwin, J.S. The effect of age on short-term outcomes after pancreatic resection: A population-based study. Ann. Surg. 2008, 248, 459-467. [CrossRef]

12. Janssen, Q.P.; Buettner, S.; Suker, M.; Beumer, B.R.; Addeo, P.; Bachellier, P.; Bahary, N.; Bekaii-Saab, T.; Bali, M.A.; Besselink, M.G.; et al. Neoadjuvant FOLFIRINOX in Patients with Borderline Resectable Pancreatic Cancer: A Systematic Review and Patient-Level Meta-Analysis. J. Natl. Cancer Inst. 2019, 111, 782-794. [CrossRef]

13. Hatzaras, I.; Schmidt, C.; Klemanski, D.; Muscarella, P.; Melvin, W.S.; Ellison, E.C.; Bloomston, M. Pancreatic Resection in the Octogenarian: A Safe Option for Pancreatic Malignancy. J. Am. Coll. Surg. 2011, 212, 373-377. [CrossRef]

14. Finlayson, E.; Fan, Z.; Birkmeyer, J.D. Outcomes in Octogenarians Undergoing High-Risk Cancer Operation: A National Study. J. Am. Coll. Surg. 2007, 205, 729-734. [CrossRef]

15. Groen, J.V.; Douwes, T.A.; Van Eycken, E.; Van Der Geest, L.G.M.; Johannesen, T.B.; Besselink, M.G.; Koerkamp, B.G.; Wilmink, J.W.; Bonsing, B.A.; on behalf of the Dutch Pancreatic Cancer Group; et al. Treatment and Survival of Elderly Patients with Stage I-II Pancreatic Cancer: A Report of the EURECCA Pancreas Consortium. Ann. Surg. Oncol. 2020, 27, 5337-5346. [CrossRef]

16. Kachare, S.D.; Liner, K.R.; Vohra, N.A.; Zervos, E.E.; Hickey, T.; Fitzgerald, T.L. Assessment of health care cost for complex surgical patients: Review of cost, re-imbursement and revenue involved in pancreatic surgery at a high-volume academic medical centre. HPB 2015, 17, 311-317. [CrossRef]

17. Wang, J.; Ma, R.; Churilov, L.; Eleftheriou, P.; Nikfarjam, M.; Christophi, C.; Weinberg, L. The cost of perioperative complications following pancreaticoduodenectomy: A systematic review. Pancreatology 2018, 18, 208-220. [CrossRef] 
18. von Elm, E.; Altman, D.G.; Egger, M.; Gøtzsche, P.C.; Vandenbroucke, J.; STROBE Initiative. The Strengthening the Reporting of Observational Studies in Epidemiology (STROBE) statement: Guidelines for reporting observational studies. Ann. Intern. Med. 2007, 147, 573-577. [CrossRef] [PubMed]

19. Weniger, M.; Moir, J.; Damm, M.; Maggino, L.; Kordes, M.; Rosendahl, J.; Ceyhan, G.O.; Schorn, S.; Schmid, D.; D’Haese, J.G.; et al. Respect-A multicenter retrospective study on preoperative chemotherapy in locally advanced and borderline resectable pancreatic cancer. Pancreatology 2020, 20, 1131-1138. [CrossRef] [PubMed]

20. Trestini, I.; Paiella, S.; Sandini, M.; Sperduti, I.; Elio, G.; Pollini, T.; Melisi, D.; Auriemma, A.; Soldà, C.; Bonaiuto, C.; et al. Prognostic Impact of Preoperative Nutritional Risk in Patients Who Undergo Surgery for Pancreatic Adenocarcinoma. Ann. Surg. Oncol. 2020, 27, 5325-5334. [CrossRef] [PubMed]

21. Keck, T.; Wellner, U.F.; Bahra, M.; Klein, F.; Sick, O.; Niedergethmann, M.; Wilhelm, T.J.; Farkas, S.A.; Börner, T.; Bruns, C.J.; et al. Pancreatogastrostomy Versus Pancreatojejunostomy for RECOnstruction After PANCreatoduodenectomy (RECOPANC, DRKS 00000767): Perioperative and Long-term Results of a Multicenter Randomized Controlled Trial. Ann. Surg. 2016, 263, 440-449. [CrossRef]

22. Yang, P.-C.; Huang, K.-W.; Pua, U.; Kim, M.-D.; Li, S.-P.; Li, X.-Y.; Liang, P.-C. Prognostic factor analysis of irreversible electroporation for locally advanced pancreatic cancer-A multi-institutional clinical study in Asia. Eur. J. Surg. Oncol. (EJSO) 2020, 46, 811-817. [CrossRef]

23. Holzgang, M.; Eigl, B.; Erdem, S.; Gloor, B.; Worni, M. Irreversible Electroporation in Pancreatic Cancer. Adv. Pancreat. Cancer 2018. [CrossRef]

24. Bassi, C.; Marchegiani, G.; Dervenis, C.; Sarr, M.; Abu Hilal, M.; Adham, M.; Allen, P.J.; Andersson, R.; Asbun, H.J.; Besselink, M.G.; et al. The 2016 update of the International Study Group (ISGPS) definition and grading of postoperative pancreatic fistula: 11 Years After. Surgery 2017, 161, 584-591. [CrossRef] [PubMed]

25. Wente, M.N.; Veit, J.A.; Bassi, C.; Dervenis, C.; Fingerhut, A.; Gouma, D.J.; Izbicki, J.R.; Neoptolemos, J.P.; Padbury, R.T.; Sarr, M.G.; et al. Postpancreatectomy hemorrhage (PPH)-An International Study Group of Pancreatic Surgery (ISGPS) definition. Surgery 2007, 142, 20-25. [CrossRef]

26. Berríos-Torres, S.I.; Umscheid, C.A.; Bratzler, D.W.; Leas, B.; Stone, E.C.; Kelz, R.R.; Reinke, C.E.; Morgan, S.; Solomkin, J.S.; Mazuski, J.E.; et al. Centers for Disease Control and Prevention Guideline for the Prevention of Surgical Site Infection, 2017. JAMA Surg. 2017, 152, 784-791. [CrossRef] [PubMed]

27. Banks, P.A.; Bollen, T.L.; Dervenis, C.; Gooszen, H.G.; Johnson, C.D.; Sarr, M.G.; Tsiotos, G.G.; Vege, S.S. Classification of acute pancreatitis-2012: Revision of the Atlanta classification and definitions by international consensus. Gut 2012, 62, 102-111. [CrossRef] [PubMed]

28. Dindo, D.; Demartines, N.; Clavien, P.A. Classification of surgical complications: A new proposal with evaluation in a cohort of 6336 patients and results of a survey. Ann. Surg. 2004, 240, 205-213. [CrossRef]

29. Bettelli, G. Preoperative evaluation of the elderly surgical patient and anesthesia challenges in the XXI century. Aging Clin. Exp. Res. 2018, 30, 229-235. [CrossRef] [PubMed]

30. Shahrokni, A.; Vishnevsky, B.M.; Jang, B.; Sarraf, S.; Alexander, K.; Kim, S.J.; Downey, R.; Afonso, A.; Korc-Grodzicki, B. Geriatric Assessment, Not ASA Physical Status, Is Associated With 6-Month Postoperative Survival in Patients with Cancer Aged >/=75 Years. J. Natl. Compr. Cancer Netw. 2019, 17, 687-694. [CrossRef]

31. Augustin, T.; Burstein, M.D.; Schneider, E.B.; Morris-Stiff, G.; Wey, J.; Chalikonda, S.; Walsh, R.M. Frailty predicts risk of life-threatening complications and mortality after pancreatic resections. Surgery 2016, 160, 987-996. [CrossRef]

32. Merath, K.; Mehta, R.; Tsilimigras, D.I.; Farooq, A.; Sahara, K.; Paredes, A.Z.; Wu, L.; Ejaz, A.; Pawlik, T.M. In-hospital Mortality Following Pancreatoduodenectomy: A Comprehensive Analysis. J. Gastrointest. Surg. 2019, 24, 1119-1126. [CrossRef]

33. De Hert, S.; Staender, S.; Fritsch, G.; Hinkelbein, J.; Afshari, A.; Bettelli, G.; Bock, M.; Chew, M.S.; Coburn, M.; de Robertis, E.; et al Pre-operative evaluation of adults undergoing elective noncardiac surgery: Updated guideline from the European Society of Anaesthesiology. Eur. J. Anaesthesiol. 2018, 35, 407-465. [CrossRef] [PubMed]

34. Biondetti, P.; Fumarola, E.M.; Ierardi, A.M.; Carrafiello, G. Bleeding complications after pancreatic surgery: Interventional radiology management. Gland. Surg. 2019, 8, 150-163. [CrossRef]

35. Akgul, O.; Merath, K.; Mehta, R.; Hyer, J.M.; Chakedis, J.; Wiemann, B.; Johnson, M.; Paredes, A.; Dillhoff, M.; Cloyd, J.M.; et al. Postoperative Pancreatic Fistula Following Pancreaticoduodenectomy-Stratification of Patient Risk. J. Gastrointest. Surg. 2019, 23, 1817-1824. [CrossRef]

36. Roberts, K.J.; Hodson, J.; Mehrzad, H.; Marudanayagam, R.; Sutcliffe, R.P.; Muiesan, P.; Isaac, J.; Bramhall, S.R.; Mirza, D.F. A preoperative predictive score of pancreatic fistula following pancreatoduodenectomy. HPB 2014, 16, 620-628. [CrossRef] [PubMed]

37. Wellner, U.F.; Kayser, G.; Lapshyn, H.; Sick, O.; Makowiec, F.; Höppner, J.; Hopt, U.T.; Keck, T. A simple scoring system based on clinical factors related to pancreatic texture predicts postoperative pancreatic fistula preoperatively. HPB 2010, 12, 696-702. [CrossRef]

38. Bicakli, D.H.; Uslu, R.; Güney, S.C.; Coker, A. The Relationship between Nutritional Status, Performance Status, and Survival among Pancreatic Cancer Patients. Nutr. Cancer 2020, 72, 202-208. [CrossRef] [PubMed]

39. Del Chiaro, M.; Rangelova, E.; Segersvard, R.; Arnelo, U. Are there still indications for total pancreatectomy? Updates Surg. 2016, 68, 257-263. [CrossRef] 
40. Mackay, T.M.; Smits, F.J.; Roos, D.; Bonsing, B.A.; Bosscha, K.; Busch, R.; Creemers, G.; van Dam, M.; van Eijck, H.J.; Gerhards, F.; et al. The risk of not receiving adjuvant chemotherapy after resection of pancreatic ductal adenocarcinoma: A nationwide analysis. HPB 2019, 22, 233-240. [CrossRef]

41. Oliveira-Cunha, M.; Malde, D.J.; Aldouri, A.; Morris-Stiff, G.; Menon, K.V.; Smith, A.M. Results of pancreatic surgery in the elderly: Is age a barrier? HPB 2013, 15, 24-30. [CrossRef] [PubMed]

42. Conroy, T.; Hammel, P.; Hebbar, M.; Ben Abdelghani, M.; Wei, A.C.; Raoul, J.-L.; Choné, L.; Francois, E.; Artru, P.; Biagi, J.J.; et al. FOLFIRINOX or Gemcitabine as Adjuvant Therapy for Pancreatic Cancer. N. Engl. J. Med. 2018, 379, 2395-2406. [CrossRef] [PubMed] 